Supplement Issue 1: International Tehran Breast Cancer Congress (TBCC9)

\title{
Comparison of the Geometric and Digital Magnification to Detect Micro-Calcification in Mammogram
}

\author{
Samira Moshiri ${ }^{1}$, Mohammad Esmaiel Fazilat Moaddeli ${ }^{2}$, Sepide \\ Sefidbakht ${ }^{2}$, Fariba Zarei ${ }^{2}$, Parysa Pishdad ${ }^{2}$, Zinabe Gholami Bardeji \\ ${ }^{2}$, VC Vani ${ }^{3}$, S. Chatterjee ${ }^{4}$, Rezvan Ravanfar Haghighi ${ }^{2,}$ \\ ${ }^{1}$ Department of Nuclear and Reactor Engineering, Islamic Azad University, Arsanjan \\ 73761168, Fars, Iran \\ ${ }^{2}$ Medical Imaging Research Center, Shiraz University of Medical Sciences, Shiraz \\ 7134845794, Iran \\ ${ }^{3}$ Department of Instrumentation and Applied Physics, Indian Institute of Science, \\ Bangalore 560012, India \\ ${ }^{4}$ AIPSN, Chemical Engineering Building (Old), Indian Institute of Science, Bangalore \\ 560012, India \\ ${ }^{*}$ Corresponding author: Rezvan Ravanfar Haghighi, Medical Imaging Research \\ Center, Shiraz University of Medical Sciences, Shiraz 7134845794, Iran. E-mail: \\ sravanfarr@gmail.com
}

DOI: $10.21859 /$ mci-supp-115

\section{Keywords:}

Digital Mammography

Micro-calcification

Geometric Magnification

Digital Zoom

\begin{abstract}
Introduction: For early diagnosis of breast cancer, definitive confirmation can be reached by identification of micro-calcifications, by mammography. This study examines the relative advantages of magnification of mammography images, obtained by (a) geometric, (b) digital zoom, for which there is still no agreement.

Materials and Methods: The study was conducted with two different digital mammography systems, having geometrical and digital magnification facilities. ACR phantom containing different sizes of $\mathrm{Al} 2 \mathrm{O} 3$ specks, to mimic micro-calcification, was used. Phantom images in contact and 1.5 X magnification modes were taken. Usual glandular dose measurements were also done. These images were assessed by 3 radiologists and three medical physicists. Model calculations and statistical analyses were used to judge the relative preferences for the two modes of magnification.

Results: It is seen that in $90 \%$ cases, detection is possible up to the 4th group of specks with $0.24 \mathrm{~mm}$, by both these above method of magnification while the AVG (average glandular dose) values measured by multi-function meter were $0.88 \mathrm{mGy}$ and $2.1 \mathrm{mGy}$ at the contact and 1.5X positions respectively.

Conclusions: We find that geometric and digital zoom are both capable to detect micro-calcifications, though the mean radiation dose for $1.5 \mathrm{X}$ geometric magnification case is nearly double of that in the digital magnification case (contact position). Thus, from radiation dose considerations, the digital magnification zoom may be preferred to geometric magnification, in digital mammography systems, though their abilities to detect micro-calcification are nearly the same.
\end{abstract}

